\title{
THE FLASH PHASE OF SOLAR FLARES: SATELLITE OBSERVATIONS OF ELECTRONS
}

\author{
R. P. LIN \\ Space Sciences Laboratory, University of California, Berkeley, Calif. 94720, U.S.A.
}

\begin{abstract}
Satellite observations of solar electrons bearing on flare particle acceleration and the generation of radio and X-ray emission are reviewed. The observations support a two stage acceleration process for electrons, one stage commonly occurring at the flare flash phase and accelerating electrons up to $\sim 100 \mathrm{keV}$, and a second stage occurring only in large proton flares and accelerating electrons up to relativistic energies. The location of the acceleration region appears to be no lower than the lower corona.

The accelerated non-relativistic electrons generate type III radio burst emission as they escape from the Sun. Direct spacecraft observations of the type III emission generated near $1 \mathrm{AU}$ and the energetic electrons, provide quantitative information on the characteristics of the electrons exciting type III emission, the production of plasma waves, and the conversion from plasma waves to electromagnetic radiation.
\end{abstract}

\section{Introduction}

In the past decade spacecraft penetrating beyond the Earth's magnetosphere have been able to directly observe the low energy particle emissions of the Sun. Low energy solar particles are very frequently emitted from the Sun, especially non-relativistic electrons (see Table I) which commonly originate in small importance 1 flares or subflares. Electrons up to $\sim 10^{8} \mathrm{eV}$ in energy have been observed (Datlowe, 1971) from larger flares. Energetic electrons appear to contain the bulk of the flare energy

TABLE I

Solar particle events in an active year

\begin{tabular}{llr}
\hline Number of solar flares $^{\mathrm{a}}$ & $\sim 16,000$ \\
Number of non-relativistic electron events $^{\mathrm{b}}$ & $\sim 400$ \\
Number of energetic proton events $^{\mathrm{b}}$ & $\sim 70$
\end{tabular}

2 Normalized for the whole Sun $=$ number observed $\times 2$.

b Normalized by cone of emission of $\sim 70^{\circ}$ for non-relativistic electron and $\sim 100^{\circ}$ for proton events.

in those flares where they are accelerated (Lin and Hudson, 1971; Syrotvatskii and Shmeleva, 1972) and they are responsible for most of the observed flare energetic X-ray and radio emission. The particle observations, combined with improved observations of solar electromagnetic radiation (much of which has also been provided by spacecraft) define a detailed physical picture of energetic electrons in small solar flares. Here we review the results obtained from direct spacecraft sampling of electrons from flares pertaining to the acceleration of electrons at the sun and to the generation of type III radio emission at $1 \mathrm{AU}$. 


\section{Interplanetary Propagation}

The electron observations at $1 \mathrm{AU}$ are for the most part limited to the particle intensity above a given energy threshold. The intensity vs time profiles for flare associated events are generally consistent with an impulsive injection into the interplanetary medium. If the electrons are scattered a great deal in their propagation from the Sun to $1 \mathrm{AU}$ (mean free path $=\lambda \ll 1 \mathrm{AU}$ ) then the intensity-time profile will look diffusive, as in Figure 1. If, on the other hand, very little scattering occurs $(\lambda \gtrsim 1 \mathrm{AU})$ then the
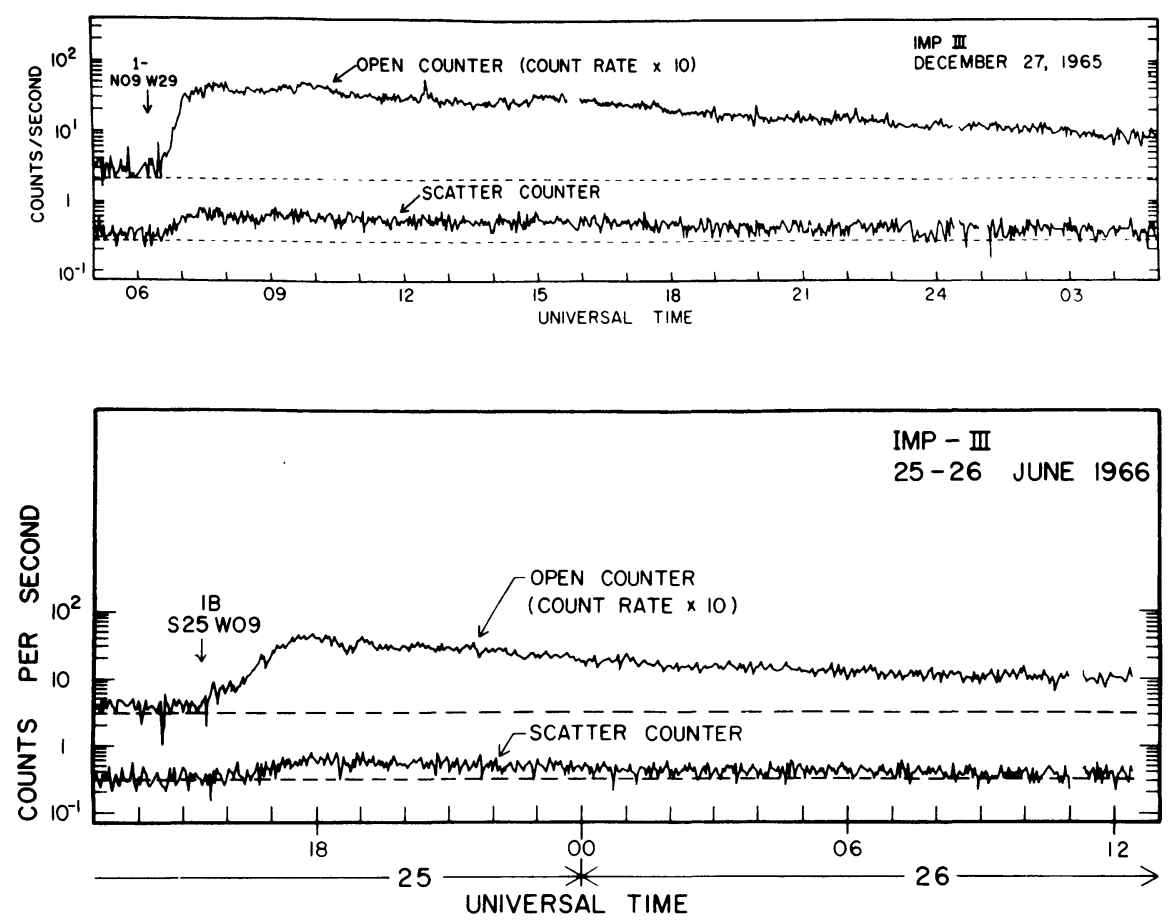

Fig. 1. Two diffusive electron events. The scatter counter is only sensitive to $>45 \mathrm{keV}$ electrons while the open counter counts both $>40 \mathrm{keV}$ electrons and $>0.5 \mathrm{MeV}$ protons. The 27 December 1965 event shows a rapid rise during onset which is observed for many electron events.

intensity-time profile will consist of a rapid increase and decrease as in Figure 2. Both kinds of events, diffusive and scatter-free (Lin, 1970), and events in between as well, are observed, with scatter-free events $(\lambda>1 \mathrm{AU})$ numbering $\sim 20 \%$ of the total.

It might seem, then, that it would be very difficult to derive the characteristics of the injected electrons since the observations at $1 \mathrm{AU}$ are greatly affected by the amount of scattering and how it varies with energy, etc. Actually, however, the maximum flux is remarkably insensitive to the details of the scattering (Lin, 1971), so long as there is enough scattering to give diffusive profiles. We can obtain the relationship between the maximum flux and the number of particles emitted for an event which can be 


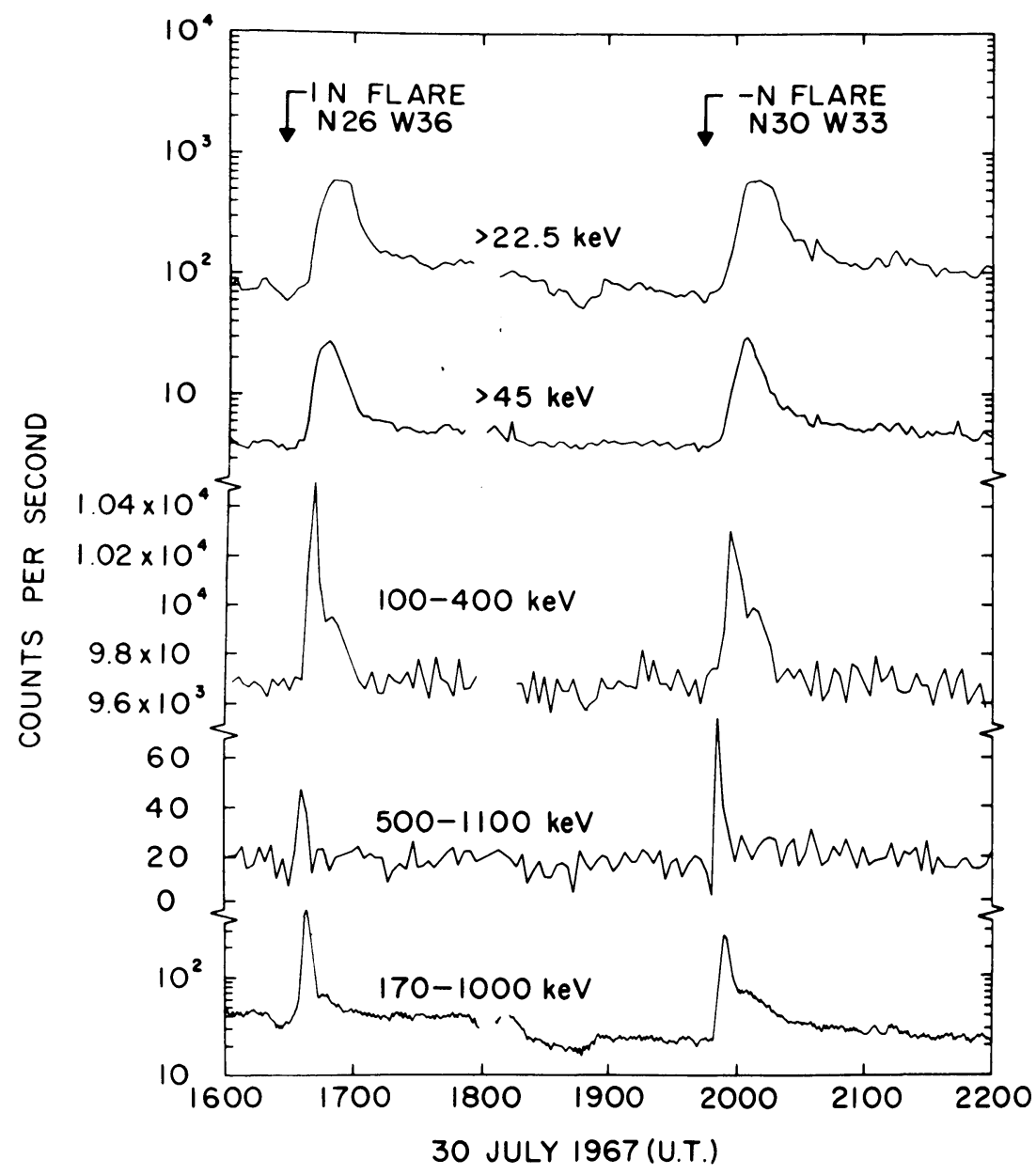

Fig. 2. Two scatter-free impulsive solar electron events from McMath plage 8905 (Wang et al., 1971). The sharp initial peak in the high energy channels place an upper limit on the duration of the electron injection into the interplanetary medium of $\$ 3 \mathrm{~min}$. Note the long decay following the initial peak in the low energy channels.

described by a diffusion equation (Parker, 1963)

$$
\frac{\partial \varrho}{\partial t}=\frac{1}{r^{\alpha}} \frac{\partial}{\partial r}\left(M r^{\alpha+\beta} \frac{\partial \varrho}{\partial r}\right),
$$

where the diffusion coefficient $D=M r^{\beta}, M$ and $\beta$ are parameters. Here $\varrho$ is the particle density at position $r$, time $t$, of energy $E$ and $\alpha$ is the parameter specifying the dimension of the space to be used. From Krimigis (1965), we obtain for an assumed isotropic flux $J=\varrho v / 4 \pi$,

$$
J(r, t)=\frac{N v \exp \left[-\frac{1}{M t} \frac{r^{2-\beta}}{(2-\beta)^{2}}\right]}{\left.4 \pi(2-\beta)^{(2 \alpha+\beta) /(2-\beta)} \Gamma[\alpha+1) /(2-\beta)\right]}\left(\frac{1}{M t}\right)^{(\alpha+1) /(2-\beta)},
$$


where $N=$ number of particles emitted per unit solid angle, and $v=$ particle velocity. At time of maximum we obtain

$$
\begin{aligned}
& J_{\max }\left(r, t_{\max }\right)= \\
& =\frac{N v \exp \left[-\frac{\alpha-1}{2-\beta}\right]}{4 \pi(2-\beta)^{(2 \alpha+\beta) /(2-\beta)} \Gamma[(\alpha+1) /(2-\beta)]}\left(\frac{(2-\beta)(\alpha+1)}{r^{2-\beta}}\right)^{(\alpha+1) /(2-\beta)} .
\end{aligned}
$$

Note that the relationship between $N$ and $J_{\max }$ is independent of $M$. Thus, as long as the diffusion coefficients for the different energy particles have the same spatial dependence (same $\beta$ ) regardless of the value of $M$, the constant of proportionality between $J_{\max }$ and $N v$ remains the same. In this case the shape of the flux spectrum derived from the maximum flux at each energy observed at $1 \mathrm{AU}$ is exactly that of the emitted particles.

Some computations have been made of the validity of this method as $\lambda$ increases (Lin et al., 1973b). These indicate that up to $\lambda \lesssim 0.3 \mathrm{AU}$ this constant relationship holds.

\section{Location of the Acceleration Region}

The electrons escaping to the interplanetary medium will lose energy during their passage through the solar atmosphere overlying the acceleration region. A straightforward calculation, assuming rectilinear upward path through fully ionized hydrogen to $1 \mathrm{AU}$ without regard to deflections or other energy loss mechanisms (such as wave particle interactions or radio emission), (Lin, 1973) shows that an initial power law spectrum at height $h$ at the Sun

$$
\frac{\mathrm{d} n}{\mathrm{~d} E_{1}}=A E_{1}^{-\delta} \quad \text { with } A, \delta \text { constants }
$$

becomes a peaked spectrum

$$
\frac{\mathrm{d} u}{\mathrm{~d} E_{2}}=\frac{A E_{2}}{\left(E_{2}^{2}+2 k\right)^{(\delta+1) / 2}}
$$

with peak at

$$
E_{2 m}=\left(\frac{2 k}{\delta}\right)^{1 / 2}
$$

and $k=-2.6 \times 10^{-18} \int_{h}^{1} \mathrm{AU} n_{i}(x) \mathrm{d} x$, where $n_{i}$ is in $\mathrm{cm}^{-3}, h$ in $\mathrm{cm}$.

In actuality the helical paths of the electrons along the field line and deflections will increase the path length traversed by the electrons in escaping to $1 \mathrm{AU}$, so that the $E_{2 m}$ given by Equation 6 will be a lower limit. A few low energy spectra of electrons observed at $1 \mathrm{AU}$ have become available from recent spacecraft observations (Figure 3). All of these spectra extend smoothly in a power law to below $\sim 6 \mathrm{keV}$, and on occasion to lower energies. Using Equation (6) we find that a peak $\lesssim 6 \mathrm{keV}$ implies 
that the total path length is given by

$$
\int_{h}^{1 \mathrm{AU}} n_{i}(x) \mathrm{d} x \lesssim 3.5 \times 10^{19} \mathrm{~cm}^{-2} .
$$

This corresponds to $\$ 60 \mu \mathrm{g} \mathrm{cm}^{-2}$ of hydrogen, equivalent to an ambient density at the acceleration region of certainly less than $\sim 10^{10} \mathrm{~cm}^{-3}$. This density corresponds to a height of $\gtrsim 2 \times 10^{4} \mathrm{~km}$ above the photosphere for a $10 \mathrm{X}$ Baumbach-Allen active region density model.

We wish to re-emphasize the fact that this estimater is a lower limit to the actual
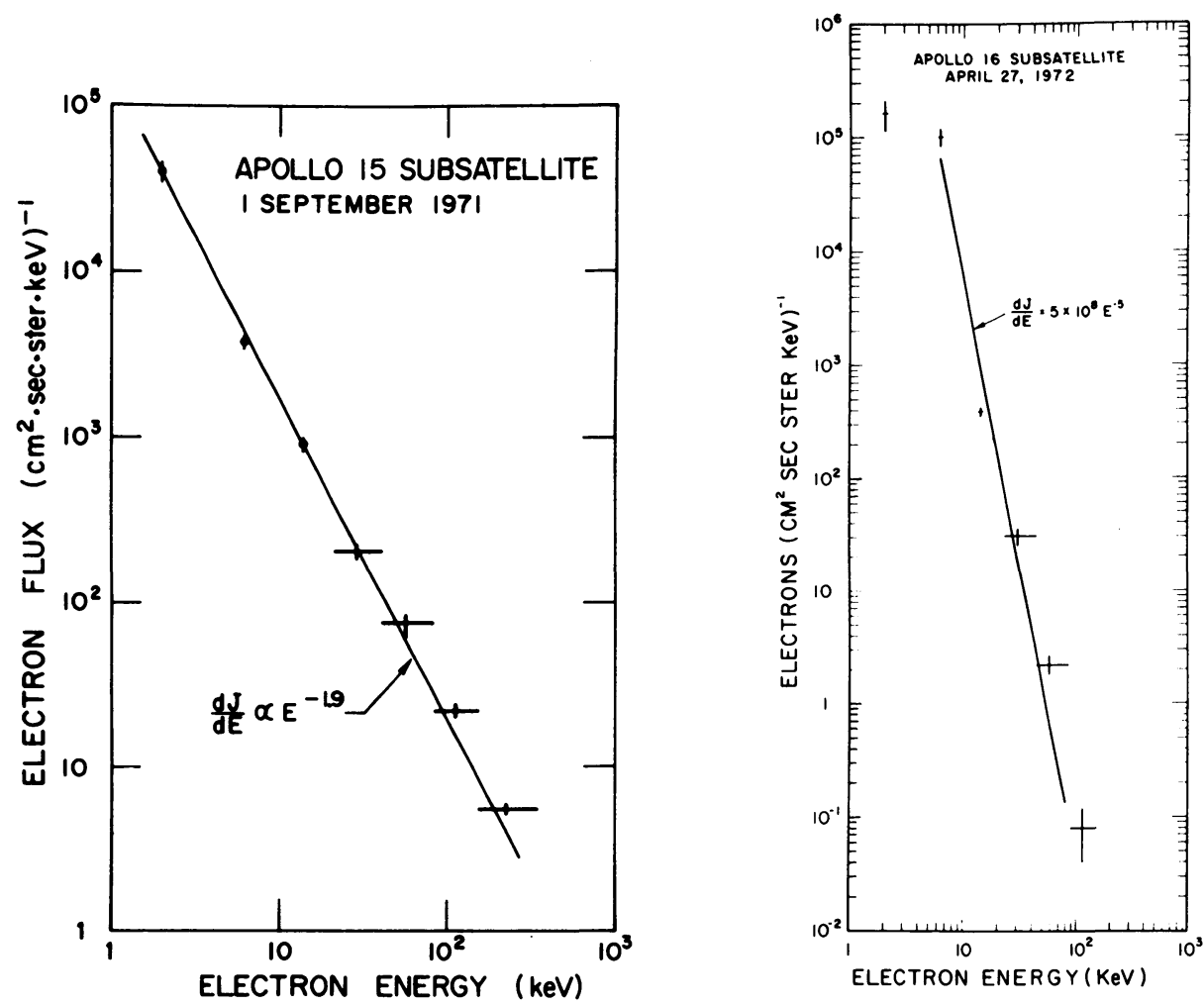

Fig. 3. Two electron energy spectra extending to low energies. The 1 September event is accompanied by energetic protons while the 27 April event (from Lin et al., 1973) is not. Both spectra extend smoothly in a power law to below $\sim 6 \mathrm{keV}$.

height of acceleration since the effects which were not taken into account would tend to increase the minimum energy of the peak. Clearly electron acceleration must have occurred in the lower corona. Although only a few events have been observed to energies below $\sim 20 \mathrm{keV}$, in no events has a turnover been observed at higher energies. Thus electron acceleration at the flash phase appears to be a coronal phenomenon, at least for events observed to emit electrons into the interplanetary medium. 
This location and ambient density is consistent with the observed starting frequencies ( $\sim 200-1000 \mathrm{MHz})$ of type III bursts, and is also consistent with the occasional observation of an electron event at $1 \mathrm{AU}$ without detectable X-ray emission (Kane and Lin, 1972). Presumably in those events the magnetic field structure in the vicinity of the acceleration region is such as to prevent the electrons from entering dense regions where a detectable X-ray flux would be produced.

\section{Accelerated vs Escaping Electron Energy Spectra}

Non-thermal X-rays give direct information on the electrons at the Sun. The X-ray spectrum can be directly related to the instantaneous X-ray producing electron spectrum (Brown, 1971; Kane and Anderson, 1970). This relationship can be written for a power law X-ray spectrum as

$$
\frac{\mathrm{d} n}{d E}=3.85 \times 10^{41} \gamma(\gamma-1)^{2} B\left(\gamma-\frac{1}{2}, \frac{3}{2}\right) \frac{A E^{-\gamma+1 / 2}}{n_{i} V} \mathrm{~cm}^{-3} \mathrm{keV}^{-1},
$$

where

$$
\frac{\mathrm{d} J(h v)}{\mathrm{d}(h v)}=A(h v)^{-v}
$$

$B(x, y)$ is the beta function, $n_{i}$ the ambient ion density, $V$ the volume of the X-ray region, and $A, \gamma$ constants.

The relationship of the instantaneous X-ray producing electron spectrom to the accelerated electron spectrum depends on the evolution of the electrons subsequent to acceleration. Suppose the electrons are accelerated in one region and produce the bulk of the observed X-rays in another region (these two regions may be one and the same but for the sake of generality we will allow them to be different). The evolution of the electron distribution, $N(E, t)=V(\mathrm{~d} n / \mathrm{d} E)$, where $V=$ volume in the X-ray emitting region, can be described by the equation

$$
\frac{\partial N(E, t)}{\partial t}=F(E, t)-\frac{N(E, t)}{\tau_{e}(E)}-\frac{\partial}{\partial E}\left[N(E, t) \frac{\mathrm{d} E}{\mathrm{~d} t}\right]
$$

where $F(E, t)$ is the input source of electrons $\mathrm{keV}^{-1} \mathrm{~s}^{-1}, N(E, t) / \tau_{e}(E)$ is the number of electrons escaping the region $\mathrm{s}^{-1} \mathrm{keV}^{-1}$, and the third term describes energy loss processes for the electrons. Note that X-ray observations define $N(E, t)$ subject to a choice of ambient density $n_{i}$ (Equation (7)). Thus this equation can be solved for $F(E, t)$, given $n_{i}$ and given the form of $\tau_{e}(E)$ if only collisional energy losses are assumed to be important.

To a good approximation we can consider $N(E, t)$ as constant over some time interval, $\Delta t$, and zero outside that interval. This removes the time dependence of the equation. Additionally we shall consider only the power law case, $N(E, t)=B E^{-\delta}$, so 
that inserting for $\mathrm{d} E / \mathrm{d} t$ the energy loss in ionized hydrogen (Trubnikov, 1965)

$$
\frac{\mathrm{d} E}{\mathrm{~d} t}=-4.9 \times 10^{-9} n_{i} E^{-1 / 2}\left(\mathrm{keV} \mathrm{s}^{-1}\right),
$$

where $n_{i}=$ ambient density in $\mathrm{cm}^{-3}$ and $E$ is in $\mathrm{keV}$, Equation (8) becomes

$$
F(E)=N(E)\left[\frac{1}{\tau_{e}(E)}+\frac{4.9 \times 10^{-9} n_{i}\left(\delta+\frac{1}{2}\right)}{E^{3 / 2}}\right] .
$$

We have computed the anticipated energy dependence of $F(E, t)$ compared to $N(E, t)$ and $\mathrm{d} J(h v) / \mathrm{d}(h v)$, and the energy dependence of the escaping electrons for two extremes:

(1) where the escape term is much larger than the collisional energy loss term. This situation is the thin-target approximation for $\mathrm{X}$-ray emission.

(2) where the collisional energy loss term is much larger than the escape term. This situation is the thick-target approximation for X-ray emission.

We have used two obvious choices for the energy dependence of $\tau_{e}$, although other forms might be appropriate. These two are: (1) $\tau_{e}=$ constant, and (2) $\tau_{e} \propto 1 / E^{1 / 2}$, i.e., proportional to the scale size of the $\mathrm{X}$-ray region divided by the particle velocity. The results are summarized in Table II.

TABLE II

\begin{tabular}{|c|c|c|}
\hline & Thick target & Thin target \\
\hline Spectrum of X-rays & $\frac{\mathrm{d} J(h v)}{\mathrm{d}(h v)}=A(h v)^{-\gamma}$ & $\frac{\mathrm{d} J(h v)}{\mathrm{d}(h v)}=A(h v)^{-\gamma}$ \\
\hline $\begin{array}{l}\text { Spectrum of electrons in X-ray } \\
\text { emitting region } N(E) \propto \frac{\mathrm{d} n e}{\mathrm{~d} E} \propto E^{-\delta}\end{array}$ & $\delta=\gamma-\frac{1}{2}$ & $\delta=\gamma-\frac{1}{2}$ \\
\hline $\begin{array}{l}\text { Spectrum of accelerated electrons } \\
F(E) \propto E^{-\delta_{a}} \\
\text { Spectrum of electrons escaping from } \\
\text { the X-ray region, } S(E) \propto E^{-\delta_{\varepsilon}}\end{array}$ & $\begin{array}{l}\delta_{a}=\gamma+1 \\
\delta_{e}=\gamma-\frac{1}{2} \text { for } \tau_{e}=\text { constant } \\
\delta_{e}=\gamma-1 \text { for } \tau_{e} \propto E^{-1 / 2}\end{array}$ & $\begin{array}{l}\delta_{a}=\gamma-\frac{1}{2} \text { for } \tau_{e}=\text { constant } \\
\delta_{a}=\gamma-1 \text { for } \tau_{e} \propto E^{-1 / 2} \\
\delta_{e}=\delta_{a}=\gamma-\frac{1}{2} \text { for } \tau_{e}=\text { constant } \\
\gamma-1 \text { for } \tau_{e} \propto E^{-1 / 2}\end{array}$ \\
\hline
\end{tabular}

Spectral dependence of electrons and $\mathrm{X}$-rays

The spectrum of electrons escaping from the X-ray region is not necessarily the spectrum of the electrons escaping to the interplanetary medium. The electrons need not escape to the interplanetary medium to be lost from the X-ray region; they may also escape to the low density, $n_{i} \leqslant 10^{9} \mathrm{~cm}^{-3}$, upper corona, where the flux of X-rays they produce will be below the threshold of current X-ray detectors. Also the acceleration region may be much higher in the solar atmosphere than the $\mathrm{X}$-ray region, and the electrons observed in space may have come directly from the accelerated population (i.e., $\delta_{e}=\delta_{a}$ ).

Datlowe and Lin (1973) noted that it is possible to distinguish between thick and thin target cases under the assumption that the spectrum of electrons observed in the 
interplanetary medium is representative of the accelerated electron spectrum (i.e., $\delta_{e}=\delta_{a}$, see Table II). For a flare event where high energy resolution measurements were available for the electrons and X-rays above $20 \mathrm{keV}$ (see Figure 4), the result was $\delta_{a}=\gamma-\frac{1}{2}$, favoring thin target. Other X-ray electron events studied where only measurements with poor energy resolution were available are also generally consistent
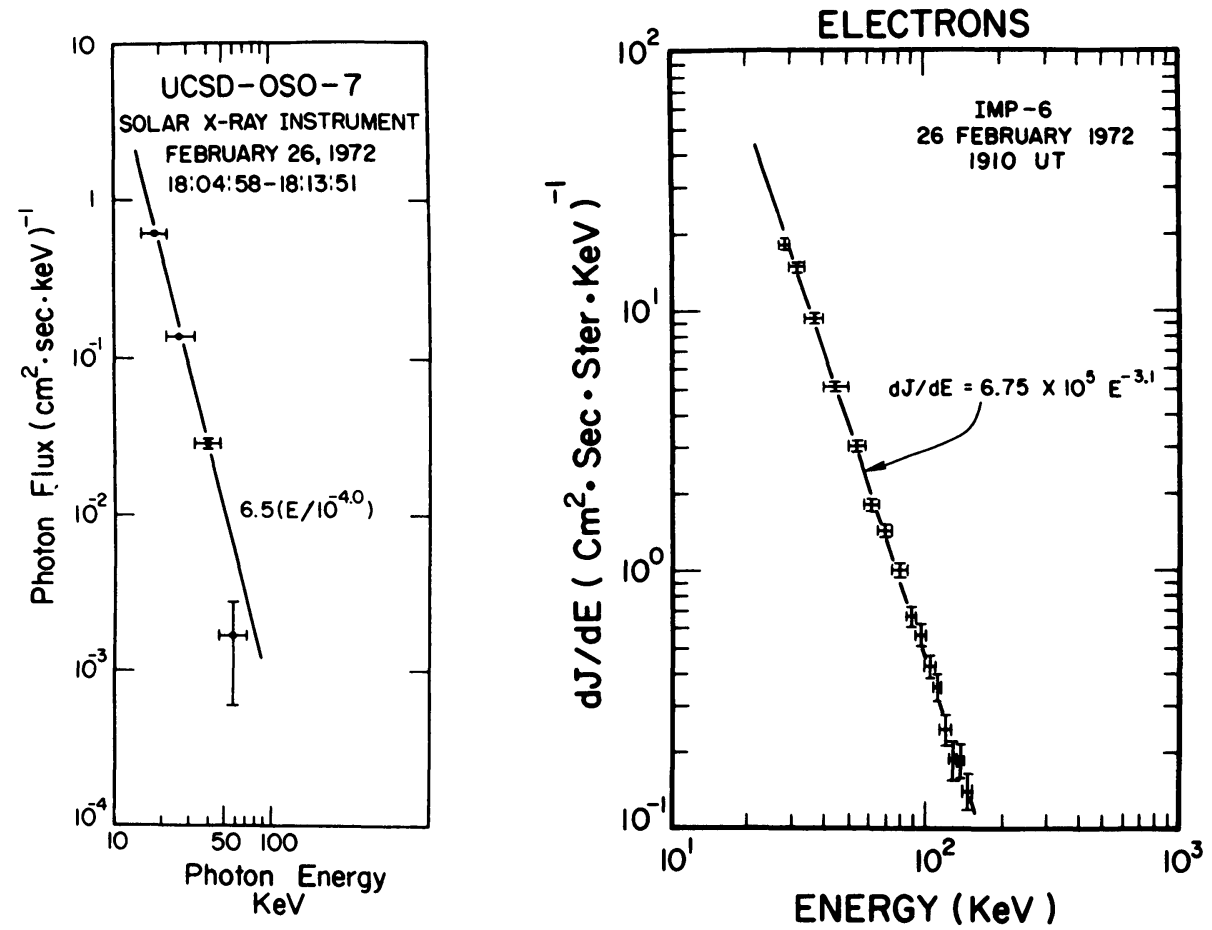

Fig. 4. The spectra of hard X-rays and electrons observed at $1 \mathrm{AU}$ for the same flare event. The photons fit a power law spectrum $\mathrm{d} J(h v) / \mathrm{d}(h v)=A(h v)^{-\gamma}$ where $\gamma=4.0 \pm 0.3$, while the electrons fit a spectrum $\mathrm{d} J / \mathrm{d} E=6.75 \times 10^{5} E^{-3.1}$. Since $\mathrm{d} n / \mathrm{d} E=v \mathrm{~d} J / \mathrm{d} E$ where $v$ is the electron velocity, the electron fit a density spectrum $\mathrm{d} n / \mathrm{d} E \propto E^{-\delta}$ with $\delta=3.6 \pm 0.1$. These two spectra are consistent with thin target emission under the assumption the escaping electrons have the same spectrum as the accelerated electrons.

(see Lin and Hudson, 1971; Kane and Lin, 1972) with a thin target model. The thin target case is also consistent with the location of the acceleration region $\left(n_{i} \lesssim 10^{10} \mathrm{~cm}^{-3}\right)$ derived from considerations of the low energy electron spectrum observed at $1 \mathrm{AU}$.

In favor of thick target processes we note that if non-relativistic electrons penetrate to the dense $\left(n_{i} \gtrsim 10^{12} \mathrm{~cm}^{-3}\right)$ regions of the chromosphere-corona boundary and below, they could produce the observed EUV and perhaps provide the energy for heating the $\mathrm{H} \alpha$ flare region through collisional loss (and possibly even heat the white light flare region) (Hudson, 1972). The close time coincidence between the hard X-ray spike and the EUV spike (Kane and Donnelly, 1971) is consistent with such an interpretation. At those densities the thick-target approximation would certainly be appropriate. 
There are several possible ways of reconciling the observations in support of thick and thin target. One possibility is that the electrons injected into the interplanetary medium may have a spectrum modified from that of the electrons initially accelerated. From comparisons of the total energy in accelerated electrons derived from the X-ray observations and the total energy in escaping electrons, Lin and Hudson (1971) found that the escape efficiency is only $\sim 0.1$ to $1 \%$. Thus the probability of escape of the electrons into the interplanetary medium may be a function of electron energy. We do not favor this possibility in view. of (a) the relatively small amounts of matter which are traversed by the escaping electrons, and (b) the otherwise coincidental agreement between the $\mathrm{X}$-ray and electron spectra.

A second possibility is that electrons of low energies, say below $\sim 10 \mathrm{keV}$, are described by the thick target approximation while higher energy electrons are in an essentially thin target situation (Kane, 1973). This dichotomy could arise, for example, if the electrons are accelerated and contained by a magnetic 'bottle' in a low density, $n_{i} \lesssim 10^{10} \mathrm{~cm}^{-3}$, region. Electrons only appear in high density, $n_{i} \gtrsim 10^{10} \mathrm{~cm}^{-3}$, regions near the feet of the magnetic bottle if they are scattered into the loss cone. Since the amount of scattering is a strongly decreasing function of energy, essentially only the low energy electrons will be dumped into the loss cone. This interpretation is consistent with the observations which show that the correspondence between rising portion of the EUV emission and the rising portion of the non-thermal X-rays is best for the lowest energy, $\sim 10 \mathrm{keV}$, X-rays.

A third possibility is that the acceleration of the escaping electrons is separate from the acceleration of the electron producing $\mathrm{X}$-rays. However the time of injection of the electrons into the interplanetary medium, which can be obtained accurately ( $\$ 10 \mathrm{~min}$ ) by analyses of the velocity dispersion observed during the onset of electron events at $1 \mathrm{AU}$, is clearly between the $\mathrm{H} \alpha$ onset and maximum, i.e., at the time of the flash phase X-ray and radio flare phenomena. The duration of the injection is inferred to be $\$ 3 \mathrm{~min}$ from the duration of the highly scatter-free events. Furthermore, escaping electrons generate type III emission. Type III emission occurs exactly at the time of X-ray bursts to within seconds when both are observed from flares (Kane, 1972).

These considerations indicate that the $>20 \mathrm{keV}$ electron spectrum observed at $1 \mathrm{AU}$ is probably an essentially undistorted sample of the electrons accelerated in the flare.

\section{Two Stage Acceleration}

Two types of electron spectra are observed (Lin, 1970). For events which are unaccompanied by energetic protons * and relativistic electrons, i.e., pure non-relativistic electron events, the electron spectrum can be fit to a power law with exponent from $\sim 2$ to 5 , usually with a steepening to $>5$ at $\sim 100-200 \mathrm{keV}$ (Figure 5). Events which are accompanied by energetic protons usually have electron spectra which extend

* Above a threshold of $\sim 0.3\left(\mathrm{~cm}^{2} \mathrm{~s} \text { ster }\right)^{-1}$ above $10 \mathrm{MeV}$. 
smoothly in a power law to relativistic energies (Figure 6). These two types of spectra, one with a 'cut-off' and one without, suggest two stages of acceleration, one a flash phase acceleration of mainly just $\sim 5-100 \mathrm{keV}$ electrons, and the second an acceleration of protons and electrons to high, even relativistic energies which occurs only in some flares. This concept is further supported by spacecraft observations which in-
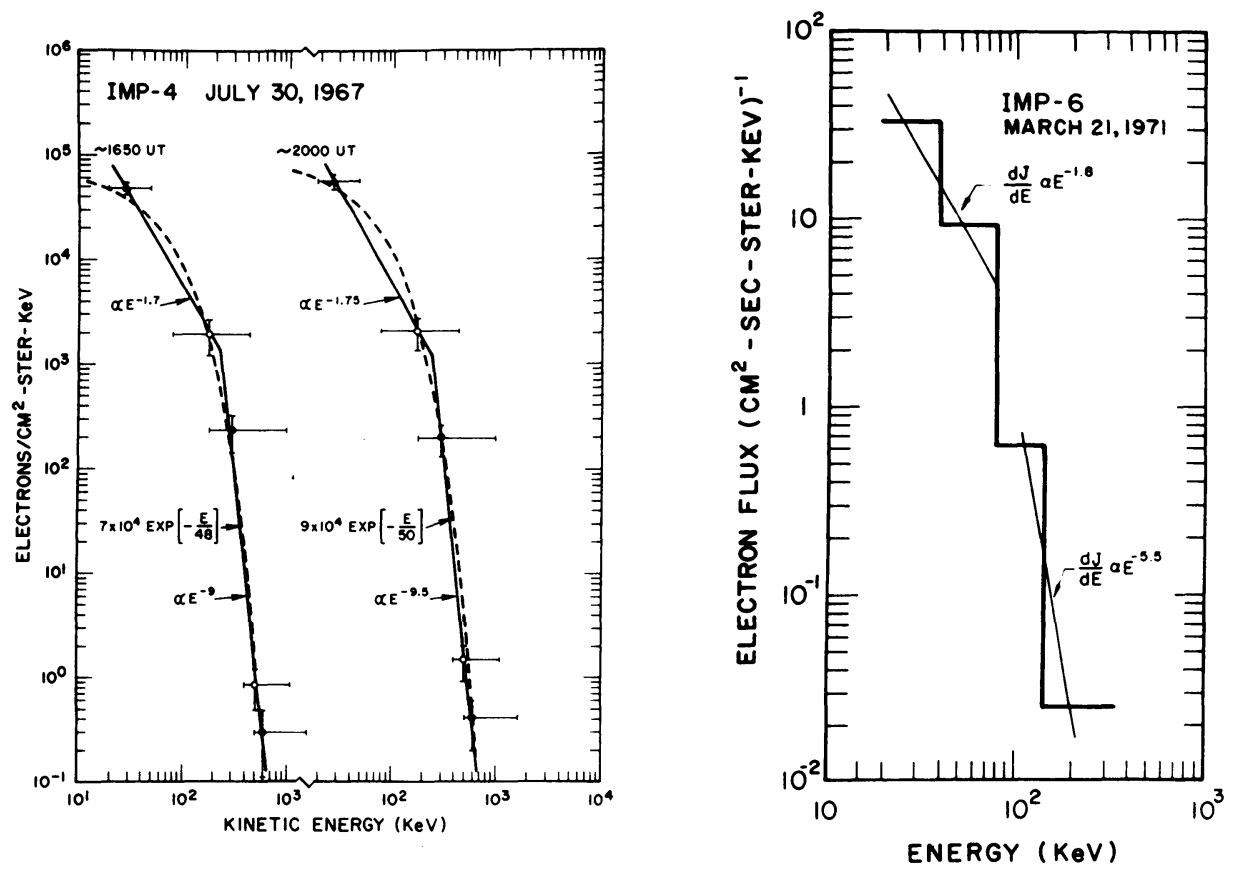

Fig. 5. The electron energy spectra of the events of Figure 2 (from Wang et al., 1971) illustrating the steepening in the spectrum above $\sim 100-200 \mathrm{keV}$ typical of pure electron events. Note the wide variation in flux from the 30 July 1967 events to the 21 March 1971 event.

dicate that relativistic electrons and energetic protons are usually injected into the interplanetary medium $\gtrsim 10 \mathrm{~min}$ after the non-relativistic electrons (Figure 7) (Sullivan, 1974; Simnett, 1974; Lin and Anderson, 1967).

Two stages are sometimes observed in the hard X-ray event accompanying energetic proton flares (Figure 8) (Frost and Dennis, 1971). The X-ray energy spectrum shows a cut-off at $\sim 100 \mathrm{keV}$ for the flash phase but no energy cut-off even to the limits of their observation $(\gtrsim 250 \mathrm{keV})$ in the long second phase. That phase starts at the onset of the type II burst. The radio, X-ray, and particle observations are generally consistent with the acceleration of particles in the second state by the type II shock front in the corona by a stochastic Fermi-type mechanism. The X-ray event of 30 March 1969

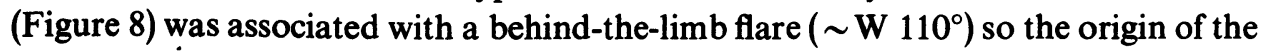
$\mathrm{X}$-ray burst must be in the corona. Interplanetary shocks and the Earth's bow shock both accelerate particles, electrons and nuclei, up to energies of $\sim 10^{2} \mathrm{keV}$ (Fan et al., 1964; Anderson, 1965; McGuire et al., 1972) and $10 \mathrm{MeV}$ (Palmeira et al., 1971) re- 


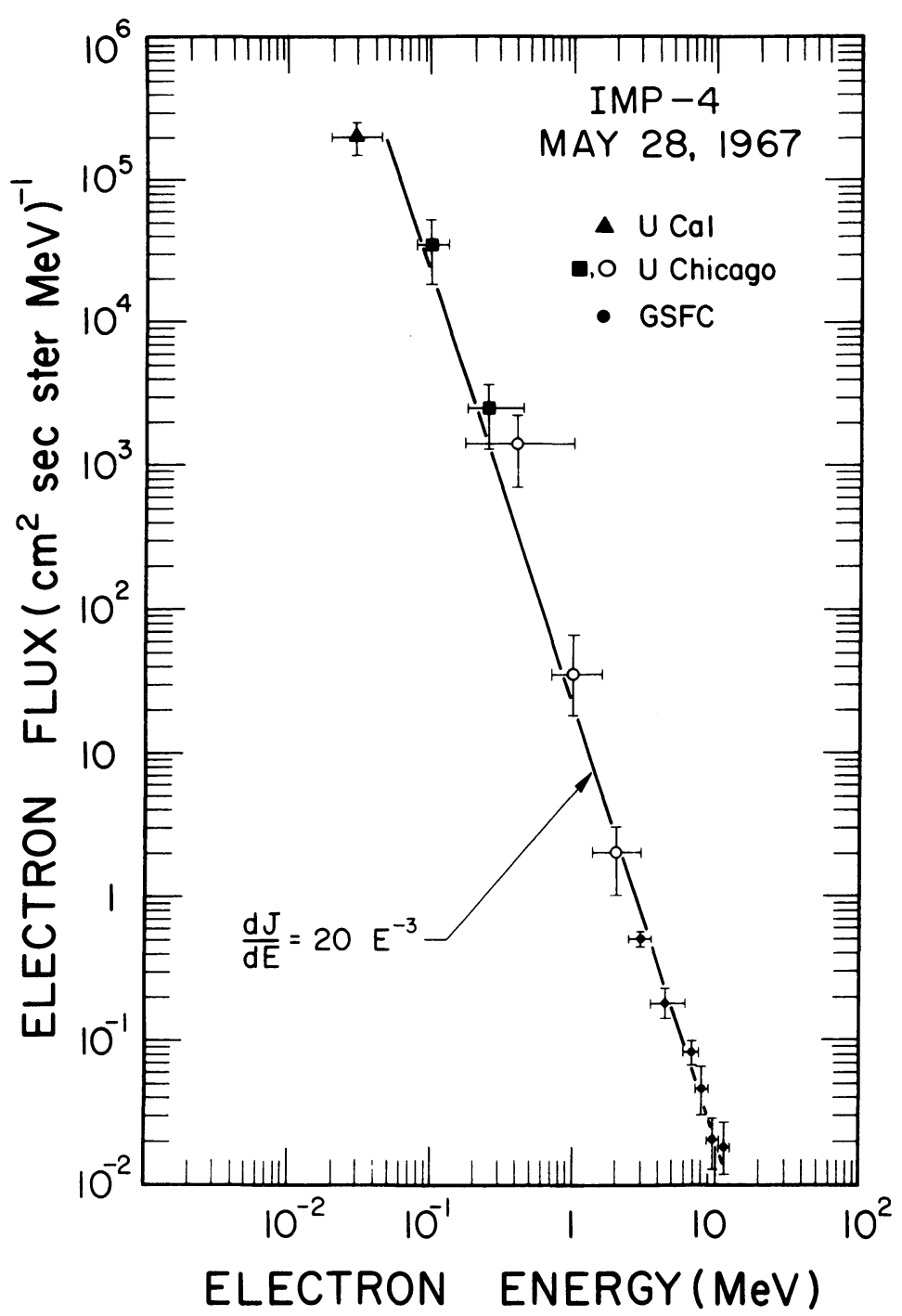

Fig. 6. The differential electron energy spectrum for the mixed electronproton event of 28 May 1967, compiled from four different detector systems aboard IMP-4. The lowest energy point is obtained from Geiger Müller detector observations (Lin, 1970). The University of Chicago points are from solid-state detector telescopes (Sullivan, 1973), and the points above $\sim 2 \mathrm{MeV}$ are from the Goddard Space Flight Center range and energy loss scintillation detectors (Simnett, 1971). The points fit to a single power over three decades in energy, even though they are from several different detectors with different view directions.

spectively. In the much higher magnetic fields and densities near the Sun it seems likely that substantially higher particle energies will be attained.

\section{Generation of Type III Radio Emission}

It is well established that energetic electrons generate most (if not all) of the non- 
thermal radio emission observed from the Sun. Detailed calculations of gyro-synchrotron and synchrotron emission of energetic electrons in solar magnetic fields give generally good agreement with observations of impulsive microwave bursts and type IV emission. However until recently the various theoretical treatment of type III emission differed as to the exciter, whether electrons or protons or waves, and numbers

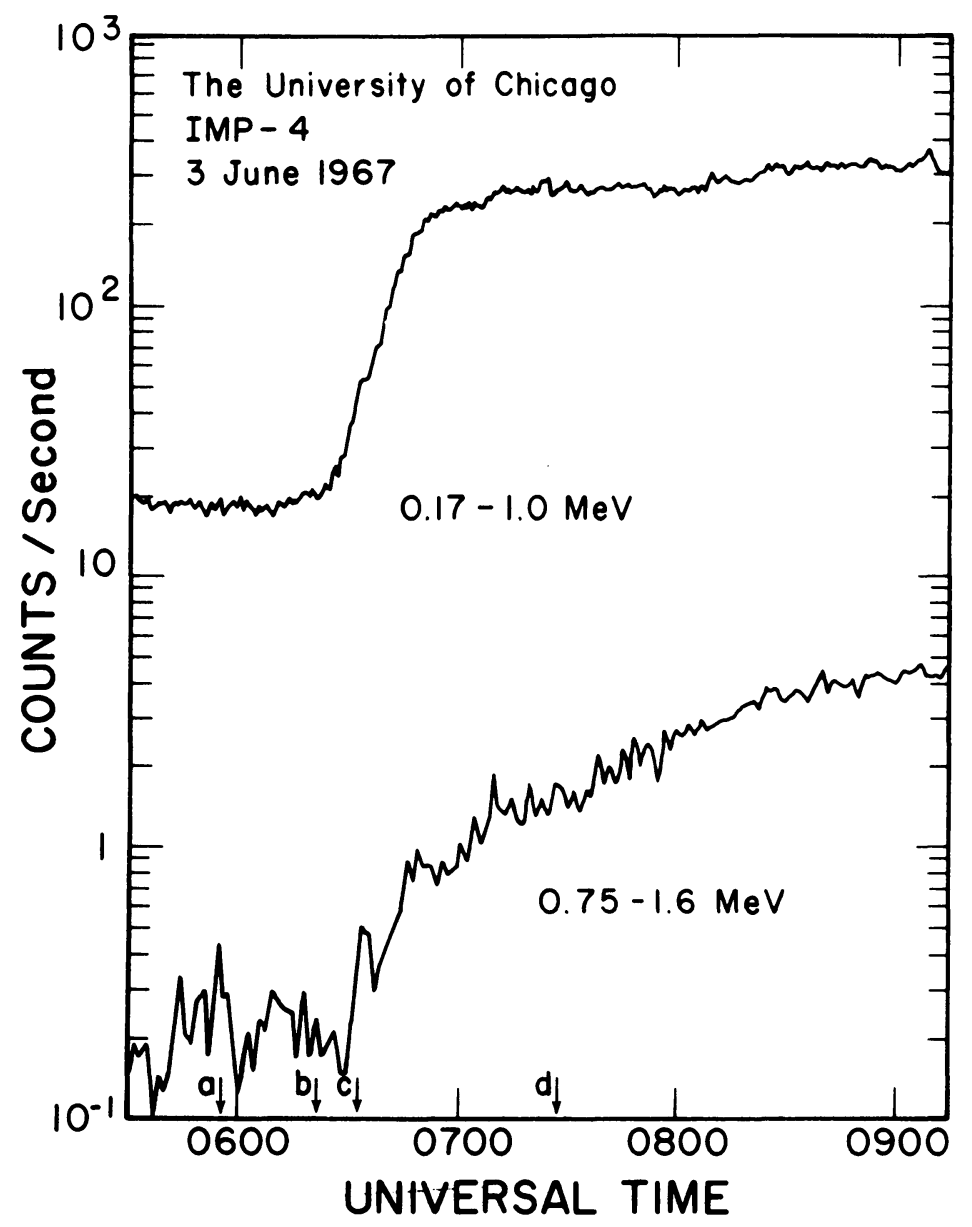

Fig. 7. The delay of the relativistic (0.75-1.6 MeV) electrons vs the non-relativistic electrons (0.17-1 MeV) is illustrated here. The onset of the soft 2-12 $\AA$ flare X-rays is denoted by (a), the $0.17-1.0 \mathrm{MeV}$ electron onset by (b), the $0.75-1.6 \mathrm{MeV}$ onset by (c), and the 9.6-18 MeV proton onset by (d)

(taken from Sullivan, 1973).

of particles needed varied over 10-12 orders of magnitude (Evans et al., 1971). Thus direct observations of the exciter, and simultaneously the type III emission generated by them at $1 \mathrm{AU}$, are of critical importance in establishing a firm theoretical base for the emission processs. 

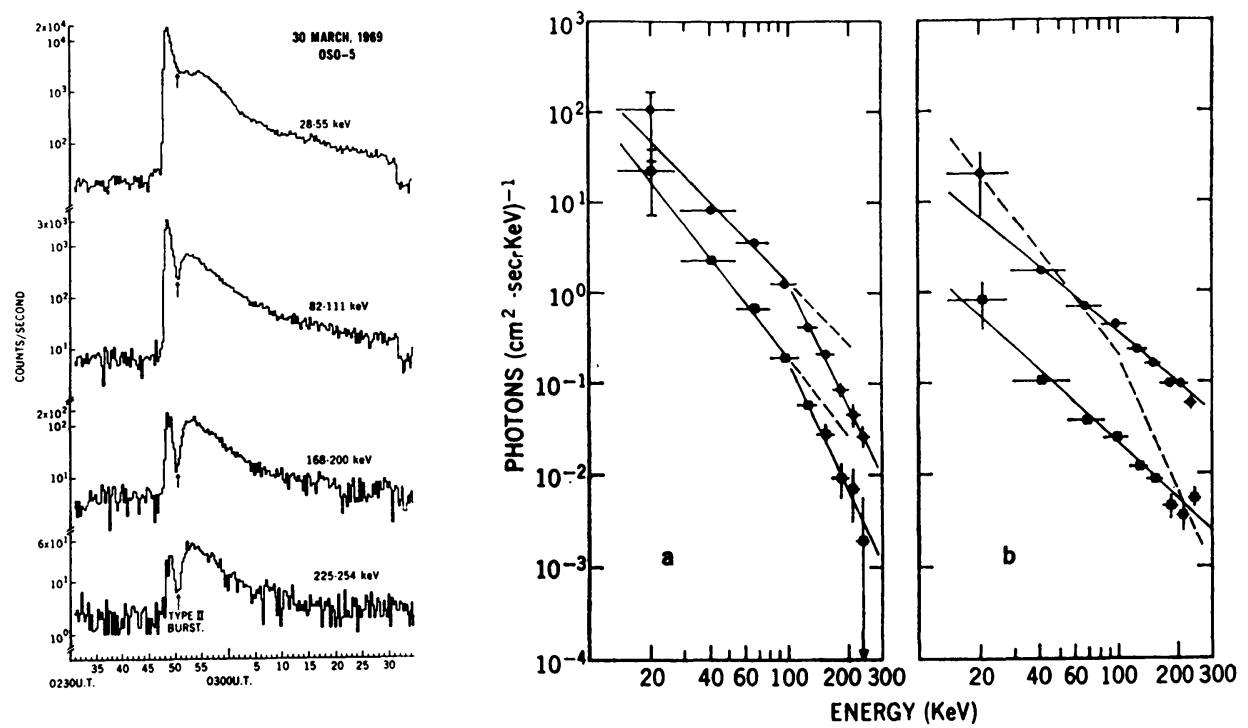

Fig. 8. The two-stage hard X-ray burst of 30 March 1969 (from Frost and Dennis, 1971), which was followed by an intense mixed electron-proton event observed at $1 \mathrm{AU}$. This $\mathrm{X}$-ray event presents clear evidence for two-stage acceleration. Photon energy spectra during the initial X-ray burst (shown in the middle panel marked ' $a$ ') have a spectrum steepening above $\sim 100 \mathrm{keV}$, fairly typical of flash phase events. The spectra during the second phase (shown in the right panel marked ' $b$ ') which starts at the time of intense type II emission (Smerd, 1970) shows a smooth and very hard spectrum to the upper limits of the X-ray detector's energy range $(\sim 300 \mathrm{keV})$.

\subsection{Physical mechanism}

Type III solar radio bursts are the most common type of impulsive phenomena observed from the Sun. These bursts are characterized by a rapid frequency drift from high to low frequencies, and occasionally by the presence of two bands of emission, one at approximately twice the frequency of the other (see reviews by Wild, et al., 1963; Wild and Smerd, 1972). A theoretical basis for the plasma hypothesis for type III solar radio bursts (Wild, 1950) was first introduced by Ginzburg and Zheleznyakov (1958), and although it has been developed and refined in the intervening years, the basic ideas have remained unchanged (see review by Smith, 1973). A group of fast particles injected near the Sun generate longitudinal electron plasma waves at frequencies near the local plasma frequency as they pass through the coronal plasma. These plasma waves then scatter off ion density fluctuations to produce electromagnetic radiation near the plasma frequency (fundamental), and off other plasma waves to produce emission at twice the plasma frequency (2nd harmonic). As the fast particles go upward in the corona and into the interplanetary medium the radio emission will drift from high to low frequencies. Typical drift rates for these bursts indicate velocities of $\sim 0.3-0.5 c$ for the particles where $c$ is the speed of light.

The plasma waves are generated through a coherent Cerenkov plasma process. In order to produce plasma waves more rapidly than they are damped, the velocity distribution of the fast particles must have a positive slope, that is, a peak must exist 
in the non-thermal particle velocity distribution. Observations of the characteristics of type III bursts and the particles that excite them will thus provide a test of basic beamplasma and mode-mode coupling theory over a wide range of plasma conditions.

\subsection{THE TYPE III BURST EXCITER}

Wild et al. (1954) were first to suggest that energetic protons might be the exciters of type III emission. More recently Smith (1970) summarized the theoretical difficulties of stabilizing a spatially unbounded and homogeneous electron stream, and noted that a proton stream can be stabilized. Smith (1970) suggested that the $\sim 20-100 \mathrm{MeV}$ protons which might produce the burst at the Sun were so few in number that after diffusion in the interplanetary medium their fluxes would be too low to observe at 1 AU. However, the observations of type III bursts generated near $1 \mathrm{AU}$ imply that substantial fluxes of protons sufficient to produce emission should be observed at $1 A U$. Such fluxes are not generally observed except in large proton events which are quite rare compared to electron events.

The theoretical difficulties for electron streams can apparently be overcome by considering a spatially bounded stream with inhomogenities in the front and back (Zaitsev et al., 1972). In addition direct observations of the electron velocity distributions at $1 \mathrm{AU}$ show that peaked distributions do exist, contrary to the theoretical predictions otherwise.

A very highly significant correlation, almost one to one, exists between intense kilometric wavelength type III's and $>20 \mathrm{keV}$ electrons observed at $1 \mathrm{AU}$ from flares located in the western solar hemisphere (Alvarez et al., 1972). In addition storms of weak type III bursts are observed at hectometric wavelengths which appear to be closely related to type I storms at metric wavelengths (Fainberg and Stone, 1970). These type III storms are accompanied by non-impulsive co-rotating $\gtrsim 20 \mathrm{keV}$ electron fluxes observed at $1 \mathrm{AU}$.

Recently Frank and Gurnett (1972) and Lin et al. (1973a) have reported observations at $1 \mathrm{AU}$ of energetic electrons and type III burst emission at the low frequencies characteristic of the near $1 \mathrm{AU}$ plasma environment. Frank and Gurnett (Figure 9) did not observe radio emission simultaneously with the arrival of the 5-6 keV electrons, which, in their interpretation, are the exciters of the emission. Rather they assumed that the radiation is generated primarily at the fundamental, i.e., local plasma frequency, and that the lowest frequency radiation they observe, $\sim 31 \mathrm{kHz}$, originates some distance away from $1 \mathrm{AU}$. Approximately $2600 \mathrm{~s}$ after the onset of the $31 \mathrm{kHz}$ emission the $\sim 6 \mathrm{keV}$ electrons are observed to arrive. Although the fundamental emission generated at $1 \mathrm{AU}(\sim 20 \mathrm{kHz})$ is not observed, the authors note that the calculated time of onset of the fundamental emission is in agreement with the arrival of the $\sim 6 \mathrm{keV}$ electrons $(v \approx 0.15 c)$. Since the drift rates of bursts near the Sun indicate velocities of $\sim 0.3 c$, they conclude that deceleration of the electrons may be substantial.

Lin et al. (1973a) located the position of the type III burst emission at each fre- 


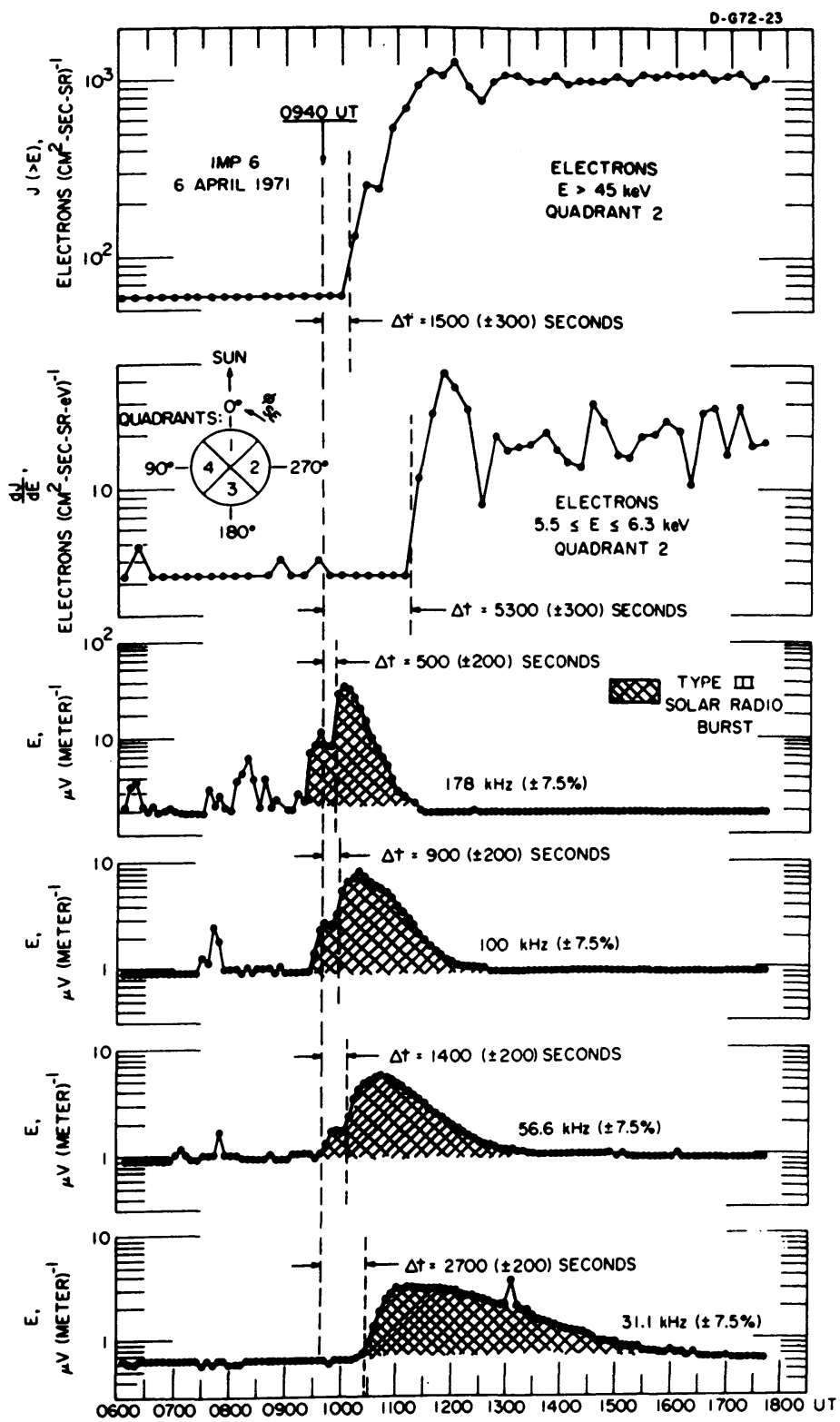

Fig. 9. Simultaneous electron and type III burst observations at 1 AU (from Frank and Gurnett, 1972). The local electron plasma frequency is $\sim 20 \mathrm{kHz}$, but no oscillations were observed at that frequency. If the radio emission is at the fundamental of the plasma frequency then the $5.5-6.3 \mathrm{keV}$ electrons would be likely candidates for the burst exciter. If, on the other hand, the radio emission is at the second harmonic of the plasma frequency then higher energy, $\gtrsim 10 \mathrm{keV}$, electrons would coincide with the emission (see text). 
quency (Figure 10) from the spin modulation of the radio signal. The emission originating at $1 \mathrm{AU}$ was then compared with the particle data. Their results show (Figure 11) that the onset of the emission located at 1 AU corresponds to the arrival of electrons of $\sim 100 \mathrm{keV}$ energy. The subsequent build up of the radiation corresponds to the arrival of lower energy electrons, until maximum is reached when $\sim 10 \mathrm{keV}$
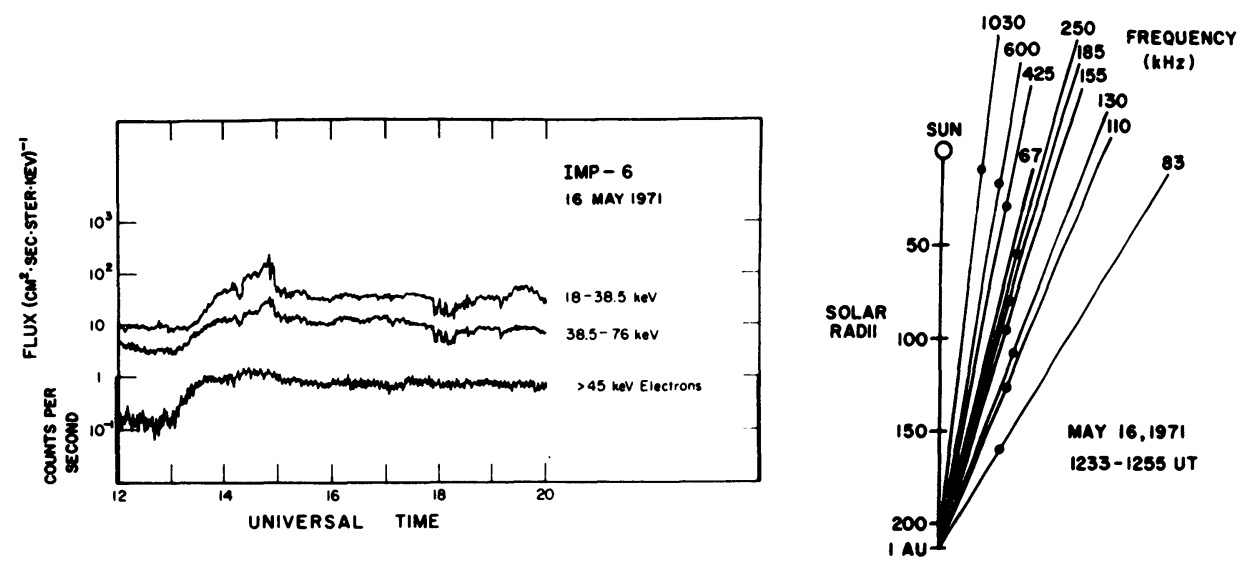

Fig. 10. (a) The May 16, 1971 event. The upper two channels are sensitive to low energy protons as well as electrons while the lower channel is sensitive only to electrons. Some upstream terrestrial protons are observed 1430-1500 UT, a time well after the period of analysis. (b) The trajectory in the interplanetary medium of the type III burst of 16 May 1971, determined from the spin modulation of the observed radio signal at different frequencies.

electrons first arrive. By the time $6 \mathrm{keV}$ electrons arrive the emission is already decaying rapidly.

Since the propagation of these electrons may differ markedly from event to event, depending on the changeable scattering characteristics of the interplanetary medium, two events were compared. One event was scatter-free, the other diffusive. The frequency drift rate of the interplanetary type III bursts was more rapid for the scatterfree event and less rapid for the diffusive event, corresponding nicely to the difference in the computed distance traveled for the first arriving electrons of $1.4 \mathrm{AU}$ in the scatter-free event and 1.7 AU for the diffusive event.

The evidence from radio studies indicate that the second harmonic emission rather than fundamental is predominant for low frequency type III radiation(Fainberg et al., 1972; Smith, 1972; Malitson et al., 1973; Haddock and Alvarez, 1973). Thus, the frequencies of the near $1 \mathrm{AU}$ radiation are $\sim$ twice the local plasma frequency. Under the assumption of second harmonic radiation the observations of Frank and Gurnett would be in close agreement with Lin et al. (1973a).

Even under the second harmonic hypothesis some apparent deceleration is observed (Fainberg et al., 1972). This can be attributed to scattering of the electrons as they propagate outward in the interplanetary medium. Such scattering will lower the apparent velocity along a smooth spiral field line. 

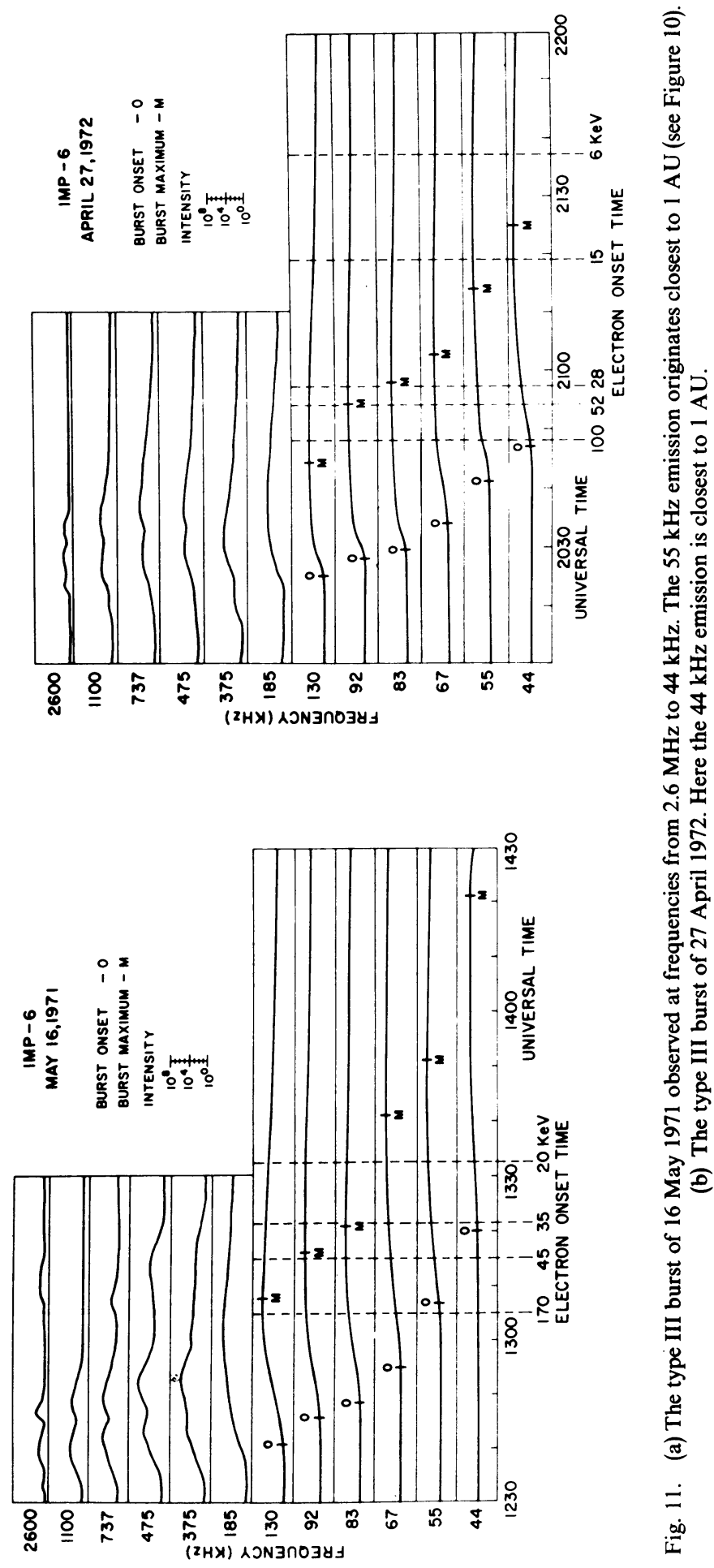

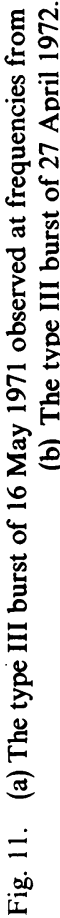




\subsection{COMPARISON WITH THEORIES}

Using calculated incoherent Cerenkov emission efficiency (Cohen, 1958) and a maximum estimated value for the efficiency of coupling to EM radiation, we find that the emission from incoherent Cerenkov processes calculated for the observed electron fluxes is insufficient to account for the observed emission.

The peaked distribution needed to generate coherent Cerenkov radiation is clearly present in the electrons (Figure 12). This peaked distribution arises from velocity
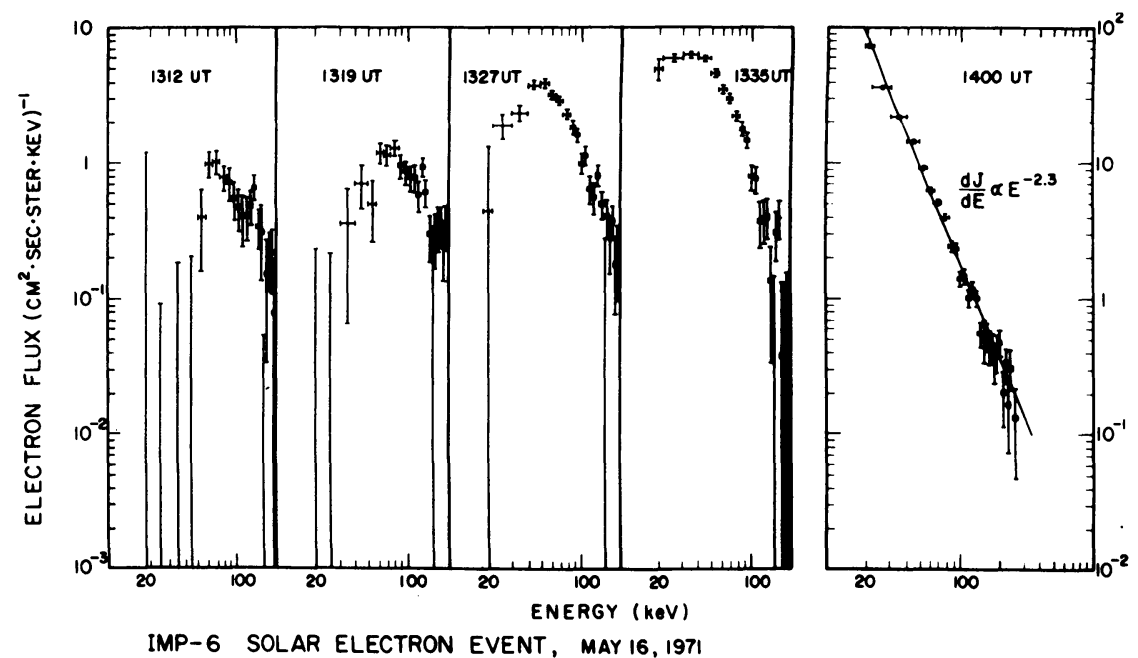

Fig. 12. The energy spectrum of the electrons in the 16 May 1971 event at different times during the onset.

dispersion rather than an initially peaked distribution of injected electrons at the Sun, since the injection spectrum at the Sun is observed to extend down to $\lesssim 5 \mathrm{keV}$ without a peak.

The radio emission is observed to increase in intensity until the peak in the electron distribution falls below $\sim 15 \mathrm{keV}$. The radio burst begins a constant exponential decay, independent of the behavior of the electron flux, after the peak in the electron distribution goes below $\sim 10 \mathrm{keV}$.

It should be noted that the coherent Celenkov processes, at least in these observations, are not so strong as to substantially modify the peaked distribution. That distribution evolves essentially as would be anticipated from velocity dispersion alone. The implication is that the time scale for relaxation of the beam through the waveparticle interaction is long compared to the time scale for evolution of the distribution by velocity dispersion. This is contrary to the expectation of most theories for type III emission.

One factor not considered in most theoretical treatments which may be relevant in explaining the long time scale for relaxation is the angular distribution of the electrons. The average distribution for the 16 May 1971 event during the period of generation of type III emission is shown in Figure 13. The distribution is not just outward 
along the field line as assumed by most theoretical models, but instead is relatively isotropic, with a maximum to minimum ratio of $\sim 2: 1$. This angular distribution presumably arises from the pitch-angle scattering of the electrons by irregularities in the interplanetary magnetic field.

Table III lists some characteristics of the low frequency type III burst at $1 \mathrm{AU}$

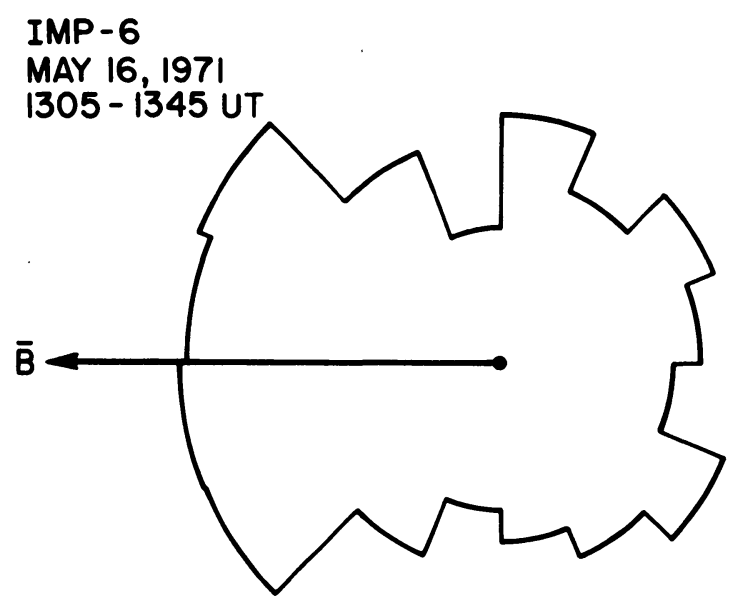

Fig. 13. The pitch angle distribution of the $>45 \mathrm{keV}$ electrons during the onset of the 16 May 1971 event.

The approximate direction of $B$ is indicated by the arrow.

\section{TABLE III}

Relevant parameters of type III bursts and burst exciters at $1 \mathrm{AU}$

(1) Type III burst
(a) Typical frequencies of observation (2nd harmonic)
$\sim 40-60 \mathrm{kHz}$
(b) Intensity of emission
$\sim 3 \times 10^{-17}$
(c) Cross-sectional area of source
$\mathrm{W} \mathrm{m}^{-2} \mathrm{~Hz}$
(d) Average emissivity of source
$\sim 10^{26} \mathrm{~cm}^{2}$
$\sim 6 \times 10^{-24} \mathrm{erg} \mathrm{cm}^{-3} \mathrm{~s}^{-1}$

(2) Fast electron exciters
(a) Typical energy of electrons
(b) Density of fast electron at burst maximum
$\sim 10-100 \mathrm{keV}$
(c) Total number of fast electrons producing emission
$\sim 10^{-6} \mathrm{~cm}^{-3}$
$\sim 10^{33}$
(d) Average rate of energy loss to electromagnetic
$\sim 4 \times 10^{-9} \mathrm{keV} \mathrm{s}^{-1}$ emission (per electron)
(e) Average interparticle distance
$\sim 10^{2} \mathrm{~cm}$

(3) Ambient plasma medium (solar wind)
(a) Mean densities
$5-10 \mathrm{~cm}^{-3}$
(b) Mean temperatures - electron
$\sim 1.2 \times 10^{5} \mathrm{~K}$
$\sim 7 \times 10^{4} \mathrm{~K}$
(c) Debye length
- proton
$\sim 10^{3} \mathrm{~cm}$ 
and some parameters of the emission process. It is clear from these preliminary studies that a quantitative plasma theory for type III bursts can be obtained through further observations of this kind. Such observations and theory, besides illuminating problems of plasma physics otherwise impossible to study, will also be applicable for the quantitative interpretation not only of solar flare radio phenomena, but possibly of galactic and extragalactic radio emission as well.

\section{Acknowledgements}

I wish to acknowledge discussions with Drs S. R. Kane and K. A. Anderson. This research was funded in part by NASA grant NGL-05-003-017.

\section{References}

Alvarez, H., Haddock, F., and Lin, R. P.: 1972, Solar Phys. 26, 468.

Anderson, K. A.: 1965, Proc. 9th International Conf. on Cosmic Rays, London, p. 520.

Brown, J. C.: 1971, Solar Phys. 18, 450.

Cohen, M. H.: 1958, Phys. Rev. 123, 711.

Datlow, D. : 1971, Solar Phys. 17, 436.

Datlowe, D. and Lin, R. P.: 1973, Solar Phys. 32, 459.

Evans, L. G., Fainberg, J., and Stone, R. G.: 1971, Solar Phys. 21, 1968.

Fainberg, J., Evans, L. G., and Stone, R. G.: 1972, Science 178, 743.

Fainberg, J. and Stone, R. G.: 1971, Astrophys. J. 164, L123.

Fan, C. Y., Gloeckler, G., and Simpson, J. A.: 1964, Phys. Rev. Letters 13, 149.

Frank, L. A. and Gurnett, D. A.: 1972, Solar Phys. $27,446$.

Frost, K. J. and Dennis, B. R.: 1971, Astrophys. J. 165, 655.

Ginzburg, V. L. and Zheleznyakov, V. V.: 1958, Soviet Astron. AJ2, 653.

Haddock, F. T. and Alvarez, H.: 1973, Solar Phys. 29, 183.

Hudson, H. S. : 1972, Solar Phys. 24, 414.

Kane, S. R.: 1972, Solar Phys. 27, 174.

Kane, S. R.: 1973, Bull. Am. Astron. Soc. 5, 274.

Kane, S. R. and Anderson, K. A.: 1970, Astrophys. J. 162, 1003.

Kane, S. R. and Donnelly, R. F.: 1971, Astrophys. J. 164, 151.

Kane, S. R. and Lin, R. P.: 1972, Solar Phys. 23, 457.

Krimigis, S. M.: 1965, J. Geophys. Res. 70, 2943.

Lin, R. P.: 1970a, J. Geophys. Res. 75, 2583.

Lin, R. P.: 1970b, Solar Phys. 12, 209.

Lin, R. P.: 1971, Conf. Papers, 12th International Conf. on Cosmic Rays, Hobart, Tasmania, Australia, 5 , 1805.

Lin, R. P.: 1973, in R. Ramaty and R. G. Stone (eds.), Proc. Symposium on High Energy Phenomena on the Sun, Goddard Space Flight Center, Greenbelt, Maryland, September 1972, p. 439.

Lin, R. P. and Anderson, K. A.: 1967, Solar Phys. 1, 446.

Lin, R. P. and Hudson, H. S.: 1971, Solar Phys. 17, 412.

Lin, R. P., Evans, L. G., and Fainberg, J.: 1973a, Astrophys. Letters 14, 191.

Lin, R. P., Wang, J. R., and Fisk, L. A.: 1974, J. Geophys. Res., in preparation.

Malitson, H. H., Fainberg, J., and Stone, R. G.: 1973, Astrophys. Letters 14, 111.

McGuire, R. E., Anderson, K. A., Chase, L. M., Lin, R. P., and McCoy, J. E. : 1972, Trans. AGU 53, 1086.

Palmeira, R. A. R., Allum, F. R., and Rao, U. R.: 1971, Solar Phys. 21, 204.

Parker, E. N.: 1963, Interplanetary Dynamical Processes, Interscience Publishers, Chapter 8.

Simnett, G. M.: 1971, Solar Phys. 20, 448.

Simnett, G. M.: 1974, Space Sci. Rev. 16, 257.

Smerd, S. F.: 1970, Proc. Astron. Soc. Australia 1, 305.

Smith, D. F.: 1970, Solar Phys. 15, 202. 
Smith, D. F.: 1972, in R. Ramaty and R. G. Stone (eds.), Proc. Symposium on High Energy Phenomena on the Sun, Goddard Space Flight Center, Greenbelt, Maryland, September, 1972, p. 558.

Sullivan, J. D. : 1974, J. Geophys. Res., to be published.

Syrovatskii, S. I. and Shmeleva, O. P.: 1972, Soviet Astron. AJ 16, 273.

Trubnikov, B. A.: 1965, Rev. Plasma Phys. 1, 105.

Wang, J. R., Fisk, L. A., and Lin, R. P.: 1971, Conf. Papers, 12th International Conference on Cosmic Rays, Hobart, Tasmania, Australia, 2, 438.

Wild, J. P.: 1950, Australian J. Sci. Res. A2, 541.

Wild, J. P., Murray, J. D., and Rowe, W. C. : 1954, Australian J. Phys. 7, 439.

Wild, J. P., Smerd, S. F., and Weiss, A. A.: 1963, Ann. Rev. Astron. Astrophys. 1, 291.

Wild, J. P. and Smerd, S. F.: 1972, Ann. Rev. Astron. Astrophys. 10, 159.

Zaitsev, V. V., Mityakov, N. A., and Rapoport, V. O.: 1972, Solar Phys. 24, 444. 


\title{
DISCUSSION
}

\author{
(After Three Review Papers by Stewart, Fainberg, and Lin)
}

Smith: What is your opinion about the constancy of the exciter speed? We heard Dr Stewart this morning saying it is constant; you wrote that there is a deceleration.

Fainberg: In the range of altitudes $50 R_{\odot}$ to the Earth's orbit, there is a deceleration, but it might not be a real variation of velocity but the result of changes in the pitch angle distribution. It is also possible that the conversion efficiency to EM waves changes with electron energy as the exciters move through different coronal regions.

Smith (to Fainberg): What do you think of the result of Haddock and Alvarez where they see the transition from the dominance of fundamental to second harmonic radiation?

Fainberg: We have specialized in simple events. The others have so many components that it is very difficult to keep track of those which go to low frequencies or not. It is always very difficult to trace individual features. Haddock and Alvarez flew their equipment on a very noisy spacecraft and had to study only strong bursts which always go in groups. Their dynamic range was limited and their events often reached saturation. I cannot have an opinion without seeing the data.

Smith (to Lin): The reason the electron stream does not relax quickly is that its density is very low relative to the background plasma so that the relaxation time is very long. The almost isotropic distribution of electrons may explain the decay of the burst below $10 \mathrm{keV}$ since any isotropic velocity distribution is stable relative to the production of plasma waves.

Dryer (to Fainberg): The IMP 6 results strongly suggest that the type III electrons may be used to map the interplanetary magnetic field. Is it possible to use this technique to map discontinuities such as shocks (emitted earlier)?

Fainberg: Yes, as long as type III's are visible. We can see that all type III's are not coming along nice spirals. But to study these disturbances systematically we would need a continuous type III output.

Sturrock: Is it true that type III's have not been correlated with the second stage of acceleration? If so, what are we to infer?

Smith: The electrons accelerated in the second phase are accelerated to relativistic energies which means that they are much more effectively stabilized by non-linear processes than lower energy electrons.

Wild: In reply to Dr Sturrock's question as to why type III's occur during the first phase of acceleration and not the second, the answer may be that, in fact, they are observed in both phases. It is possible that second phase acceleration is caused by the interaction of the type II shock wave with magnetic flux tubes. In fact, one quite often sees type III's or type III-like features (herringbone effect) emanating from type II bursts.

Pneuman (to Fainberg): The type II observations you showed in your movie seemed to show emission only in front of the disturbance propagated outward. Now if the type II burst is produced by a hydromagnetic shock moving out from the flare site, this shock wave would be expected to move out more or less in all directions. Then one should expect to see a sort of spherical geometry. Do you have any explanation as to why this does not seem to be the case?

Fainberg: The reason is that in the movie we show only the centroid of the emitting region. You cannot infer the size of this region from these pictures.

Zirin (to Lin): You said you do not see any Coulomb scattering effects but only $10^{-3}$ of the particles arrive near the Earth. Where do the other electrons go?

Lin: Presumably most of the energetic electrons are trapped high in the corona at ambient densities too low to produce observable levels of hard X-ray emission and only $\sim 1 \%$ of the electrons escape to be observed at $1 \mathrm{AU}$.

Rosenberg (to Stewart): We have been told that only harmonics are observed at low frequencies. What is the ratio of the number of fundamental to harmonic bursts in ground-based observations?

Stewart: I cannot quote numbers. It is very difficult to see pairs.

Rosenberg (to Fainberg): You showed a slide with the rise and decay times of low frequency type III showing a constant ratio. The same is true also for higher frequencies and the curve might be extended to $\sim 200 \mathrm{MHz}$ as noted by De Groot many years ago.

Schmidt (to Fainberg): Have you tried to correlate the shape of the spiral pattern followed by type III's in the range of altitudes 0 to $50 R_{\odot}$ with the velocity of solar wind?

Fainberg: We looked for that, but we do not have enough resolution to see the spiral pattern in the range $0-50 R_{\odot}$; but the technique is in principle capable of doing that. 
Erickson (to Fainberg): You stated that the motion picture and slides gave only the position of the centroid of type III emission. Could you give an estimate of the actual size of the emitting region at, say, 0.5 AU?

Fainberg: About 20 to $30^{\circ}$ as seen from the Sun.

Wild (to Sturrock): One of the important points raised this morning is the doubt cast on the more or less accepted doctrine that type III electrons travel along neutral sheets - doubt cast by the paper of Smith and Pneuman - which says that this is impossible because of the existence of transverse fields. Perhaps Dr Sturrock would care to give a second opinion on this conclusion.

Sturrock: It is certainly a problem to reconcile these conflicting views. However, I think that reconnection of field lines could produce type III electrons most easily if current sheets are very thin. If this is so, the filling out of these current sheets would allow free outflow of electrons and this could resolve the paradox.

Rosenberg: We have redone the same calculations as Pneuman and Smith. We find a much smaller transverse magnetic field than they do.

Smith: We have also redone these computations and found the same result as we published. We said that electrons cannot travel on the axis of a streamer for very long but they can travel there for $\frac{1}{2} R_{\odot}$ - then go out and remain close to the axis.

Pneuman (to Rosenberg): Any computation of a model neutral sheet relies, of course, upon the value of the electrical conductivity in the corona about which very little is known. Other considerations lead us to believe that the conductivity is considerably lower than one would normally estimate. If this is the case, then the transverse field would be even larger than either you or I would estimate. My second point is that we must clearly differentiate distinct parts of a streamer configuration - that associated with the density enhancement and the very much smaller neutral sheet in the center of the streamer. Thus, the fact that electrons cannot travel in the sheet does not exclude the possibility that streamers could be the location of type III emission, i.e. the electrons could travel outside the sheet but still in the streamer.

Stewart (to Kane): Can Dr Kane say at what altitude electrons can be accelerated by shock waves?

Kane: The observations I presented are not related to the acceleration of electrons in shock waves. The observations do indicate that during the flash phase the acceleration of electrons occurs at an altitude where the ion density is about $10^{9} \mathrm{~cm}^{-3}$. 